\title{
Delayed Initial Radioactive Iodine Therapy Resulted in Poor Survival in Patients with Metastatic Differentiated Thyroid Carcinoma: A Retrospective Statistical Analysis of 198 Cases
}

\author{
Tatsuya Higashi ${ }^{1,2}$, Ryuichi Nishii ${ }^{1}$, Shigeki Yamada ${ }^{3,4}$, Yuji Nakamoto ${ }^{2}$, Koichi Ishizu ${ }^{2}$, Shigeto Kawase ${ }^{2}$, \\ Kaori Togashi ${ }^{2}$, Satoshi Itasaka ${ }^{5}$, Masahiro Hiraoka ${ }^{5}$, Takashi Misaki ${ }^{2,6}$, and Junji Konishi ${ }^{2,7}$ \\ ${ }^{1}$ Shiga Medical Center Research Institute, Moriyama City, Shiga, Japan; ${ }^{2}$ Department of Diagnostic Imaging and Nuclear Medicine, \\ Kyoto University Graduate School of Medicine, Sakyo-ku, Kyoto, Japan; ${ }^{3}$ Department of Neurosurgery, Shiga Medical Center, \\ Moriyama City, Shiga, Japan; ${ }^{4}$ Department of Neurosurgery, Kyoto University Graduate School of Medicine, Sakyo-ku, Kyoto, Japan; \\ ${ }^{5}$ Department of Radiation Oncology and Image-Applied Therapy, Kyoto University Graduate School of Medicine, Sakyo-ku, Kyoto, \\ Japan; ${ }^{6}$ Tenri Hospital Radioisotope Center, Tenri, Nara, Japan; and ${ }^{7}$ Sugita Genpaku Memorial Obama Municipal Hospital, Obama, \\ Fukui, Japan
}

To elucidate the prognostic role of ${ }^{131}$ I radioactive iodine therapy (RIT), we conducted a retrospective cohort study analyzing the clinical factors that affect survival of postoperative patients with differentiated thyroid carcinoma (DTC). Methods: We included 198 DTC patients with extrathyroidal extension who received total or near-total thyroidectomy and then RIT in our hospital from January 1997 to June 2009: patients with lymph node metastases only ( $n=47)$, lung metastases without bone metastases $(n=105)$, or bone metastases and other distant metastases (lung, liver, brain, and skin) $(n=46)$. Hemithyroidectomy or subtotal thyroidectomy had been performed before total or near-total thyroidectomy in 59 patients. Diseasespecific survival after initial RIT was statistically evaluated using relevant clinical parameters, including age at initial RIT, pathology, sex, therapeutic history before initial RIT, pre- and posttherapeutic serum thyroglobulin ("prethyroglobulin" and "postthyroglobulin," respectively) at initial RIT, thyroglobulin under thyroid-stimulating hormone stimulation at initial RIT ("peak thyroglobulin"), grade of uptake at scintigraphy, extent of metastasis, and number of total RITs. Results: During followup after initial RIT (average, $5.37 \mathrm{y}$ ), 24 patients died from DTC (11 male patients and 13 female). The most common reasons for death were respiratory failure due to lung metastases $(n=$ 11) and uncontrollable brain metastases $(n=6)$. Univariate analysis showed that disease-specific survival was related to the following factors: extent of metastasis, age at initial RIT $(<45 \mathrm{y})$, prethyroglobulin $(<125 \mathrm{ng} / \mathrm{mL})$, peak thyroglobulin $(<1,000 \mathrm{ng} / \mathrm{mL})$, and interval from total thyroidectomy to initial RIT ( $<180 \mathrm{~d}$ ). A past history of hemithyroidectomy or subtotal thyroidectomy was not related to disease-specific survival. Multivariate analysis showed 3 factors to be independent prognostic factors-grade of ${ }^{131} \mathrm{I}$ uptake at whole-body scintigraphy, extent of metastasis, and interval to RIT ( $P \leq 0.001,0.010$, and 0.005 , respectively) - and also showed that risk of death in patients with an interval over $180 \mathrm{~d}$ was 4.22 times higher

\footnotetext{
Received Jul. 8, 2010; revision accepted Sep. 8, 2010.

For correspondence or reprints contact: Tatsuya Higashi, Shiga Medical Center Research Institute, 5-4-30, Moriyama, Moriyama City, Shiga, 5246034 Japan.

E-mail: higashi@shigamed.jp

COPYRIGHT @ 2011 by the Society of Nuclear Medicine, Inc.
}

than in those with an interval within $180 \mathrm{~d}$. Kaplan-Meier analysis revealed that a shorter interval $(180,365$, or $1,000 \mathrm{~d})$ had prognostic value even in the subgroups $45 \mathrm{y}$ or older, with lung metastases, and with bone metastases or more. Conclusion: The present study suggests that delaying initial RIT until more than $180 \mathrm{~d}$ after total thyroidectomy may result in poor survival for DTC patients.

Key Words: thyroid cancer; prognosis; radioactive iodine therapy

J Nucl Med 2011; 52:683-689

DOI: 10.2967/jnumed.110.081059

\section{D} ifferentiated thyroid carcinoma (DTC) is one of the most common endocrine tumors, and its basic treatment strategy is established $(1,2)$. Total or near-total thyroidectomy is the standard initial therapy and usually results in successful prognosis; therefore, in most countries DTC represents less than $1 \%$ of cancer deaths annually (3). However, a certain proportion of patients needs to receive additional therapy after total thyroidectomy. ${ }^{131} \mathrm{I}$ radioactive iodine therapy (RIT) is performed (sometimes palliatively) for DTC with distant or unresectable metastases and as a postoperative adjuvant for high-risk DTC with or without metastases to decrease the risk of recurrence. In general, the medical indications for RIT are considered to be as follows: presence of distant metastases, incomplete tumor resection, and complete excision of tumor but high risk for mortality or recurrence $(1,2)$. Several factors have been proposed to be of high risk, such as localized but large tumors, nonlocalized lesions with extracapsular invasion, and lymph node metastases in the neck or other sites. However, these factors are still controversial (3).

Recently, the lack of medical facilities for RIT has become one of the most important medical issues in some European countries and in Japan, because in these countries 
RIT is performed in limited numbers of medical facilities associated with strict regulation of radioactive substances (4). In Japan, the clinical situation for RIT is critical. The number of RITs increased 44\% from 2002 through 2007, although the number of available beds for RIT decreased $16 \%$ (5). This situation is serious for patients with distant metastases, and all possible courses of action from the medical and socioeconomic points of view should be taken to solve this problem.

The purpose of this study was to investigate the prognostic role of ${ }^{131}$ I RIT using a retrospective cohort study analyzing the clinical factors that affect survival after RIT for postoperative patients with DTC.

\section{MATERIALS AND METHODS}

\section{Study Population and Patient Characteristics}

From January 1997 to June 2009, 413 patients with thyroid carcinoma who received total or near-total thyroidectomy (hereinafter called total thyroidectomy) in our hospital $(n=45)$ or in other hospitals $(n=368)$ were referred and admitted to our unit in Kyoto University Hospital to receive ${ }^{131}$ I RIT. We defined 4 inclusion criteria for the present study. The first was the presence of pathologically confirmed DTC or DTC with a poorly differentiated component or other variants of DTC at the time of total thyroidectomy. The second criterion was at least one treatment by RIT at our unit from January 1997 to June 2009. The third criterion was clinical follow-up at our outpatient clinic for more than $1 \mathrm{y}$. The fourth criterion was 1 of 2 alternatives: a patient younger than $45 \mathrm{y}$ who had distant metastases at the time of thyroidectomy (stage II, based on the criteria of the International Union Against Cancer [UICC]), or a patient older than $45 \mathrm{y}$ who was in at least stage III at the time of thyroidectomy. Before RIT, the clinical stage of DTC was analyzed on the basis of the criteria of the UICC using clinical information and several imaging modalities (neck ultrasound, plain CT, or PET if available) (O). Patients were excluded if they had pathologically confirmed anaplastic carcinoma, undifferentiated carcinoma, medullary carcinoma, or malignant lymphoma at the time of total thyroidectomy; if their clinical follow-up in our outpatient clinic had been less than $1 \mathrm{y}$ or they had incomplete or missing data; or if they had an antithyroglobulin antibody level greater than $5.0 \mathrm{U} / \mathrm{mL}$.

Of the 413 patients with thyroid carcinoma, 215 were excluded because of these criteria (the criteria overlapped in some cases). No patient was pathologically confirmed as having anaplastic carcinoma, undifferentiated carcinoma, medullary carcinoma, or malignant lymphoma. Some patients $(n=145)$ had follow-up periods of less than $1 \mathrm{y}$ (most patients had no metastasis at the time of total thyroidectomy and then received RIT ablation). No patient was younger than $45 \mathrm{y}$ and in stage I at the time of thyroidectomy and received RIT. No patient was older than $45 \mathrm{y}$ and in stage I or II at the time of thyroidectomy and received RIT. Thirty patients were $45 \mathrm{y}$ old or older, of unknown clinical stage at the time of thyroidectomy, and showed postoperative serum thyroglobulin consistently lower than $5 \mathrm{ng} / \mathrm{mL}$ and peak thyroglobulin lower than $20 \mathrm{ng} / \mathrm{mL}$ under thyroid hormone withdrawal. Fortyeight patients had an antithyroglobulin antibody level greater than 5.0 U/mL. The 198 patients with DTC (total number of RITs, 598) who were finally included in the study all had extrathyroidal extension or metastasis before initial RIT. Their characteristics are shown in Table 1.
Before admission to our unit, each patient gave written informed consent to becoming a possible candidate for retrospective clinical research, as required by the Kyoto University Human Study Committee. After receiving all clinical information about RIT, including radiation exposure, each patient agreed to undergo RIT, and the patient's agreement was recorded in the patient chart (there was no special written informed consent form for RIT, because it was a part of routine clinical treatment). This study was not submitted to the ethics committee for approval, because the study relied on information obtained as part of routine clinical work.

\section{Procedures for RIT and Other Treatment}

The average interval between total thyroidectomy and initial RIT was $2.59 \pm 4.77$ y $(844 \pm 1,546 \mathrm{~d})$. The timing of referral of new patients to our unit was determined by referring surgeons, whereas decisions on initial RIT were made by our unit using a first-come, first-served policy.

${ }^{131}$ I was commercially available and provided by Fujifilm RI Pharma Co., Ltd. Initial RIT was performed using a ${ }^{131}$ I dose of $3,430 \pm 999 \mathrm{MBq}$ for a patient with resected or residual lymph node metastases, 4,292 $\pm 818 \mathrm{MBq}$ for a patient with lung metastases, and 4,677 $\pm 659 \mathrm{MBq}$ for a patient with bone metastases or more (including liver, brain, skin, or other sites).

Starting 4 wk before the planned RIT, patients withdrew from 1T4 therapy and began 50-75 $\mu \mathrm{g}$ of liothyronine (1-T3) per day for $1 \mathrm{wk}$, and they then withdrew from 1-T3 until $4 \mathrm{~d}$ after the administration of ${ }^{131} \mathrm{I}$. Recombinant human thyroid-stimulating hormone (TSH) was not used. Patients were also asked to refrain from iodine-rich foods, not to undergo contrast-enhanced CT, and to follow a low-iodine diet $(<200 \mu \mathrm{g}$ per day) for $3 \mathrm{wk}$ before RIT until $4 \mathrm{~d}$ after the administration of ${ }^{131} \mathrm{I}$. Hospital admission is mandatory in Japan for RIT in patients with DTC, because the use of more than $500 \mathrm{MBq}$ of ${ }^{131} \mathrm{I}$ on an outpatient basis is prohibited. Therefore, from the first day of RIT, patients had to stay in our medical ward-which was specially designed for the isolation of patients who had received ${ }^{131} \mathrm{I}$-for at least $3 \mathrm{~d}$, until the residual radioactivity in the body decreased to $500 \mathrm{MBq}$ or less. Then, whole-body scintigraphy (WBS) was performed to examine the biodistribution of ${ }^{131} \mathrm{I}$.

\section{Procedure for ${ }^{131}$ I WBS}

Three days ( $6 \mathrm{~d}$ in some cases) after oral administration of ${ }^{131} \mathrm{I}$, a standard WBS study (from toes to top of head) was performed using a whole-body moving-camera technique (anterior and posterior). Dual-head $\gamma$-cameras were used (either an RC-2500IV [Hitachi Medical Co.] or a Bodyscan [Siemens Medical Systems]). The scan speed was $15 \mathrm{~cm} / \mathrm{min}$ for the former camera and $10 \mathrm{~cm} / \mathrm{min}$ for the latter one, and the matrix size was $256 \times$ 1,024 pixels for both cameras. WBS images were acquired in planar view with a pair of appropriate window levels (dark and bright) and printed as hard-copy films. When a positive finding was suspected, an oblique view or SPECT images (axial and coronal) were obtained additionally using the RC-2500IV, and hardcopy films were printed. WBS images were interpreted by 1 or 2 experienced nuclear medicine physicians who were shown all available clinical information, including serum thyroglobulin level. At posttherapeutic WBS, the ${ }^{131}$ I uptake was evaluated using a 4-grade system in which 0 is excellent, 1 is good, 2 is fair, and 3 is poor. 
TABLE 1

Baseline Characteristics of All Patients, Disease-Specific Survivors, and Deceased Patients

\begin{tabular}{|c|c|c|c|c|}
\hline Characteristic & All patients $(n=198)$ & Survivors $(n=174)$ & Decedents $(n=24)$ & $P$ \\
\hline \multicolumn{5}{|l|}{ Number of RITs } \\
\hline 1 & $71(36 \%)$ & 65 (37\%) & $6(25 \%)$ & \\
\hline $2-5$ & 99 (50\%) & 86 (49\%) & $13(54 \%)$ & \\
\hline $6-9$ & $22(11 \%)$ & $19(11 \%)$ & $3(13 \%)$ & \\
\hline$\geq 10$ & $6(3 \%)$ & $4(2 \%)$ & $2(8 \%)$ & 0.397 \\
\hline Average & 3.0 & 2.9 & 4.0 & 0.361 \\
\hline Range & $1-26$ & $1-17$ & $1-26$ & \\
\hline \multicolumn{5}{|l|}{ Follow-up period (y) } \\
\hline Mean & 5.37 & 5.17 & 6.78 & 0.578 \\
\hline Range & $0.14-31.74$ & $1.13-26.84$ & $0.14-31.74$ & \\
\hline \multicolumn{5}{|l|}{ Age at initial RIT } \\
\hline Mean $(y)$ & 54.0 & 52.8 & 62.5 & 0.009 \\
\hline Range (y) & $7-84$ & $7-83$ & $48-84$ & \\
\hline$<45$ y old: $\geq 45$ y old $(n)$ & $48: 150$ (24\%:76\%) & $48: 126(28 \%: 72 \%)$ & 0:24 (0\%:100\%) & $<0.001$ \\
\hline Male:female $(n)$ & $77: 121$ (39\%:61\%) & $66: 108$ (38\%:62\%) & $11: 13$ (46\%:54\%) & 0.460 \\
\hline \multicolumn{5}{|l|}{ Initial ${ }^{131}$ I-RIT dose (MBq) } \\
\hline Mean & $3,770.3$ & $3,740.7$ & $3,988.6$ & 0.150 \\
\hline Range & $296-5,994$ & $1,665-5,994$ & $296-5,994$ & \\
\hline Pathology (PAC:FAC:other) & $\begin{array}{c}164: 28: 6 \\
\text { (83\%:14\%:3\%) }\end{array}$ & $\begin{array}{c}144: 24: 6 \\
\text { (83\%:14\%:3\%) }\end{array}$ & $\begin{array}{c}20: 4: 0 \\
\text { (83\%:17\%:0\%) }\end{array}$ & 0.644 \\
\hline \multicolumn{5}{|l|}{ WBS uptake at initial RIT (n) } \\
\hline Excellent: 0 & $117(59 \%)$ & $105(60 \%)$ & $12(50 \%)$ & \\
\hline Good: 1 & $28(14 \%)$ & $26(15 \%)$ & $2(8 \%)$ & \\
\hline Fair: 2 & $51(26 \%)$ & $42(24 \%)$ & $9(38 \%)$ & \\
\hline Poor: 3 & $2(1 \%)$ & $1(1 \%)$ & $1(4 \%)$ & 0.245 \\
\hline \multicolumn{5}{|l|}{ Metastasis extent at initial RIT (n) } \\
\hline $1^{*}$ & $47(24 \%)$ & 47 (27\%) & $0(0 \%)$ & \\
\hline $2^{\dagger}$ & $105(53 \%)$ & $92(53 \%)$ & $13(54 \%)$ & \\
\hline 3 or $4^{\ddagger}$ & $46(23 \%)$ & $35(20 \%)$ & $11(46 \%)$ & $<0.001$ \\
\hline \multicolumn{5}{|l|}{ Total thyroidectomy to initial RIT } \\
\hline Mean $(y)$ & 2.56 & 2.35 & 4.08 & 0.029 \\
\hline Range (y) & $0.07-30.98$ & $0.07-27.87$ & $0.11-30.98$ & \\
\hline$<180 \mathrm{~d}: \geq 180 \mathrm{~d}(n)$ & $106: 92$ (54\%:46\%) & $99: 75$ (57\%:43\%) & $7: 17$ (30\%:71\%) & $<0.001$ \\
\hline \multicolumn{5}{|l|}{ Previous surgery } \\
\hline Hemithyroidectomy/subtotal thyroidectomy & $59(30 \%)$ & $54(31 \%)$ & $5(21 \%)$ & \\
\hline No hemithyroidectomy/subtotal thyroidectomy & $124(63 \%)$ & $110(63 \%)$ & $14(58 \%)$ & \\
\hline Unknown & $15(8 \%)$ & $10(6 \%)$ & $5(21 \%)$ & 0.060 \\
\hline Years from hemithyroidectomy/subtotal to total & 6.3 & 5.9 & 10.2 & 0.878 \\
\hline \multicolumn{5}{|l|}{ Therapy between initial thyroidectomy and initial RIT } \\
\hline Local resection, lymph node resection, radiation, etc. & $42(21 \%)$ & $39(22 \%)$ & $3(13 \%)$ & \\
\hline No therapy & $142(72 \%)$ & $125(72 \%)$ & $17(71 \%)$ & \\
\hline Unknown & $14(7 \%)$ & $10(6 \%)$ & $4(17 \%)$ & 0.146 \\
\hline \multicolumn{5}{|l|}{ Longest blank period } \\
\hline Treated within $180 \mathrm{~d}$ & 67 (34\%) & $62(36 \%)$ & $5(21 \%)$ & \\
\hline Treated within 180-364 d & $20(10 \%)$ & $17(10 \%)$ & $3(13 \%)$ & \\
\hline $1-2 y$ & $11(6 \%)$ & $11(6 \%)$ & $0(0 \%)$ & \\
\hline $2-3 y$ & $13(7 \%)$ & $12(7 \%)$ & $1(4 \%)$ & \\
\hline$\geq 3$ y & $73(37 \%)$ & $62(36 \%)$ & $11(46 \%)$ & \\
\hline Unknown & $14(7 \%)$ & $10(6 \%)$ & $4(17 \%)$ & 0.152 \\
\hline \multicolumn{5}{|l|}{ Prethyroglobulin at initial RIT (ng/mL) } \\
\hline Mean & 1,612 & 1,493 & 2,579 & $<0.001$ \\
\hline Range & $3.0-46,386$ & $3.0-464,386$ & $160.4-10,109$ & \\
\hline \multicolumn{5}{|l|}{ Peak thyroglobulin at initial RIT (ng/mL) } \\
\hline Mean & 7,667 & 7,415 & 10,001 & $<0.001$ \\
\hline Range & $0.1-232,453$ & $0.1-232,453$ & $543-36,086$ & \\
\hline \multicolumn{5}{|l|}{ Postthyroglobulin at initial RIT (ng/mL) } \\
\hline Mean & 1,178 & 1,130 & 1,616 & $<0.001$ \\
\hline Range & $0.1-34,461$ & $0.1-34,461$ & $40.8-9,365$ & \\
\hline
\end{tabular}

*Local disease or lymph node metastases only.

${ }^{\dagger}$ Lung metastases with or without lymph node metastases.

‡Bone metastases or more.

$\mathrm{FAC}=$ follicular adenocarcinoma; mets $=$ metastases; PAC $=$ papillary adenocarcinoma. 


\section{Evaluation of Therapeutic Effect and Clinical Follow-up}

As a tumor marker, serum thyroglobulin was evaluated before each RIT ("prethyroglobulin"), at the time of RIT ("peak thyroglobulin"), and after RIT ("postthyroglobulin"). Prethyroglobulin was defined as the highest value obtained $1-3$ mo before each RIT under the TSH-suppressed condition, when serum TSH level was less than $5 \mu \mathrm{IU} / \mathrm{mL}$ (average, $1.53 \pm 1.84 \mu \mathrm{IU} / \mathrm{mL}$ ). Peak thyroglobulin was defined as the value obtained $0-1 \mathrm{~d}$ before RIT under the TSH-stimulated condition, when serum TSH level was more than $20 \mu \mathrm{IU} / \mathrm{mL}$ (average, $95.46 \pm 65.78 \mu \mathrm{IU} / \mathrm{mL}$ ). Postthyroglobulin was defined as the lowest value obtained 2-12 mo after RIT under the TSH-suppressed condition, when serum TSH level was less than $5 \mu \mathrm{IU} / \mathrm{mL}$ (average, $1.10 \pm 1.67 \mu \mathrm{IU} / \mathrm{mL}$ ).

Disease-specific survival was confirmed in patient records by clinical follow-up. The primary endpoint of survival was the date of disease-specific death. Cases were censored at the date of nondisease-specific death and at the last date of follow-up (regardless of whether the patient was lost to follow-up or completed all followup). The starting point for survival was the date of initial RIT.

\section{Statistics}

All values are expressed as mean $\pm \mathrm{SD}$. All statistical analysis was performed using R software (version 2.9.0; R Foundation for Statistical Computing) and confirmed using JMP software (version $8 \mathrm{~J}$; SAS Institute). A $P$ value of less than 0.05 was considered statistically significant in each analysis. Missing data were treated as deficit data not changing other variables (Supplemental Data; supplemental materials are available online only at http://jnm. snmjournals.org).

\section{RESULTS}

\section{Patient Characteristics}

Although 124 patients received total thyroidectomy at the time of initial detection of DTC, 59 patients had received hemithyroidectomy or subtotal thyroidectomy at first and then total thyroidectomy later (average interval, $6.3 \pm 6.1 \mathrm{y}$; interval unknown in 15 patients). In 42 patients, there was a history of other treatment (e.g., resection of local recurrence, resection of cervical lymph node recurrence, resection of lobectomy for lung metastasis, and radiation therapy of brain or bone metastasis) in the interval between initial thyroidectomy (hemithyroidectomy, subtotal thyroidectomy, or total thyroidectomy) and initial RIT, whereas initial RIT was performed after total thyroidectomy without other therapeutic procedures in 142 patients. In the interval between initial thyroidectomy and initial RIT, the lengths of the observation periods without therapy ("blank periods") were determined and the longest such period for each patient was classified as occurring within $180 \mathrm{~d}(n=67), 180-365 \mathrm{~d}$ $(n=20), 1-2 \mathrm{y}(n=11), 2-3 \mathrm{y}(n=13)$, or $3 \mathrm{y}$ or more $(n=73)$. In most patients with more than a $3-y$ therapeutic blank period ( $n=73$ ), recurrent lesions were newly detected lung or bone metastases $(n=61)$. Therapeutic procedures were performed immediately after each other.

\section{Clinical Follow-up}

Thirty-one patients died during the observation periods. Two patients who died from cachexia and respiratory failure due to lung metastases were excluded from the present study because of a high serum anti-thyroglobulin antibody value. Five patients who died for non-diseasespecific reasons (esophageal cancer, gallbladder cancer, gastric cancer, renal failure due to diabetes, and suicide) were censored in the present study. Thus, 24 deceased patients were examined by statistical analysis (11 male patients and 13 female). The reasons for death were respiratory failure due to lung metastases $(n=11)$, uncontrollable brain metastases $(n=6)$, massive hemoptysis from respiratory tract metastases $(n=3)$, asphyxia related to a bulky neck mass $(n=2)$, cachexia due to multiple metastases $(n=1)$, and hemorrhagic cardiac tamponade due to direct invasion $(n=1)$. Table 1 compares survivors and deceased patients. The average cumulative number of RITs for deceased patients was $4.0 \pm 5.2$ (range, 1-26). Age at initial RIT for deceased patients was $62.5 \pm 8.6 \mathrm{y}$ (range, 48-84 y), which was significantly greater than that of survivors $(P=0.009)$. There were no deceased patients younger than $45 \mathrm{y}$ old at the time of initial RIT and no deceased patients with only local disease or lymph node metastases at the time of initial RIT. The average interval between total thyroidectomy and initial RIT in deceased patients was $4.08 \mathrm{y}$, which was significantly longer than that of survivors $(2.38 \mathrm{y})(P=0.029)$. Factors related to other therapy before total thyroidectomy (past history of hemithyroidectomy or subtotal thyroidectomy, past history of other treatment, and longest blank period) did not statistically differ between survivors and deceased patients. Serum thyroglobulin levels (prethyroglobulin, peak thyroglobulin, and postthyroglobulin) were significantly higher in deceased patients.

\section{Univariate Analysis of Each Prognostic Factor}

Univariate analysis using 2- or 3-way segmentation was performed (Supplemental Data). Of the factors analyzed, age at initial RIT ( $\geq 45$ y old) showed a significantly high prognostic value. Kaplan-Meier analysis also showed a similar result (Fig. 1). Therefore, age-adjusted hazard ratio was also examined in the other factors. Grade of WBS and extent of metastases showed a significant difference, whereas there was no significant difference in the pathology of DTC. Tumor markers (prethyroglobulin, peak thyroglobulin, and postthyroglobulin) showed significant prognostic value both in total examinations and in age-adjusted examinations. Interval between total thyroidectomy and initial RIT (180 d or more) showed significant prognostic value $(P<0.001$, age-adjusted $P=0.012)$. Kaplan-Meier analysis also showed similar results using $180 \mathrm{~d}$ and other intervals (<365 d, <1,000 d) (Fig. 2).

\section{Multivariate Analysis of Prognostic Factors}

According to the univariate analysis, 6 significant factors (grade of WBS at initial RIT [fair or poor], extent of metastasis at initial RIT [bone metastasis or more], prethyroglobulin at initial RIT $[\geq 125 \mathrm{ng} / \mathrm{mL}]$, peak 

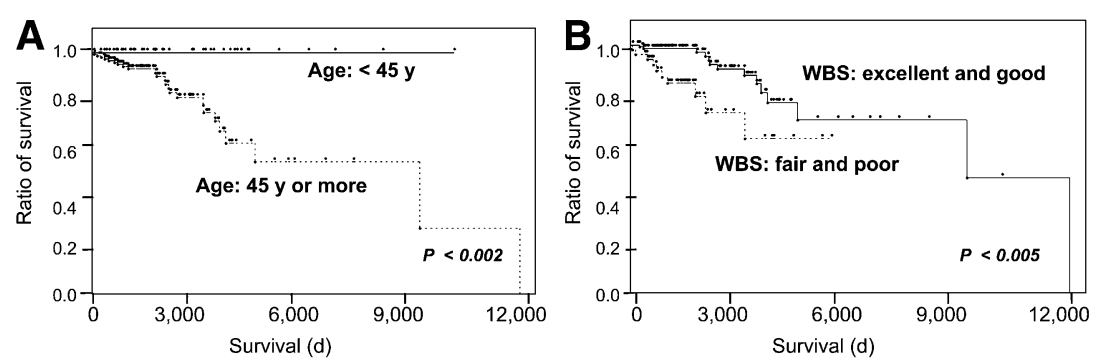

FIGURE 1. Survival curves of all DTC patients analyzed by Kaplan-Meier life tables. (A) Disease-specific survival was significantly higher in group younger than $45 \mathrm{y}$ than in group 45 y or older. (B) Disease-specific survival was significantly higher in group with better grade (excellent or good) of 131। uptake at WBS at time of initial RIT than in group with worse grade (fair or poor). (C) Disease-specific survival was significantly higher in group with low thyroglobulin before RIT $(<125 \mathrm{ng} / \mathrm{mL})$ than in group with high thyroglobulin ( $\geq 125 \mathrm{ng} / \mathrm{mL}$ ) before RIT. (D) Disease-specific survival was significantly higher in group with lymph node metastasis only than in group with lung metastasis with or without lymph node metastasis or in group with bone metastasis or more. Extent of metastasis was evaluated at time of initial RIT. LN = lymph node; mets = metastases; $\mathrm{Tg}=$ thyroglobulin.

thyroglobulin at initial RIT $[\geq 1,000 \mathrm{ng} / \mathrm{mL}]$, postthyroglobulin at initial RIT [ $\geq 500 \mathrm{ng} / \mathrm{mL}$ ], and interval between total thyroidectomy and initial RIT [ $\geq 180 \mathrm{~d}]$ ) were selected for the multivariate analysis using the stratified Cox proportional hazards model in all patients and in patients $45 \mathrm{y}$ or older at the time of initial RIT (Table 2). Grade of ${ }^{131} \mathrm{I}$ uptake at WBS after initial RIT and extent of metastasis showed significant prognostic value in all patients $(P<$ 0.001 and $P=0.010$, respectively). Interval between total thyroidectomy and initial RIT also showed significant prognostic value $(P=0.005)$. Tumor markers, prethyroglobulin, peak thyroglobulin, and postthyroglobulin did not show significant prognostic value. Analysis of patients $45 \mathrm{y}$ or older showed similar results for all these factors.
Additional data are available online (Supplemental Tables 1-3 and Supplemental Fig. 1).

\section{DISCUSSION}

A principal result of the present study is the importance of early initial RIT after total thyroidectomy. Multivariate analysis, shown in Table 2, revealed 3 independent prognostic factors: grade of ${ }^{131}$ I uptake at WBS after initial RIT, extent of metastasis at initial RIT, and interval between total thyroidectomy and initial RIT both in the entire group and in the age-adjusted group over $45 \mathrm{y}$ old. Concerning the interval between total thyroidectomy and RIT, 3 thresholds (180, 365, and 1,000 d after total thyroidectomy) showed a significant difference, but there was no significance in the analysis using $75 \mathrm{~d}$ as the threshold (Supplemental Table 2).
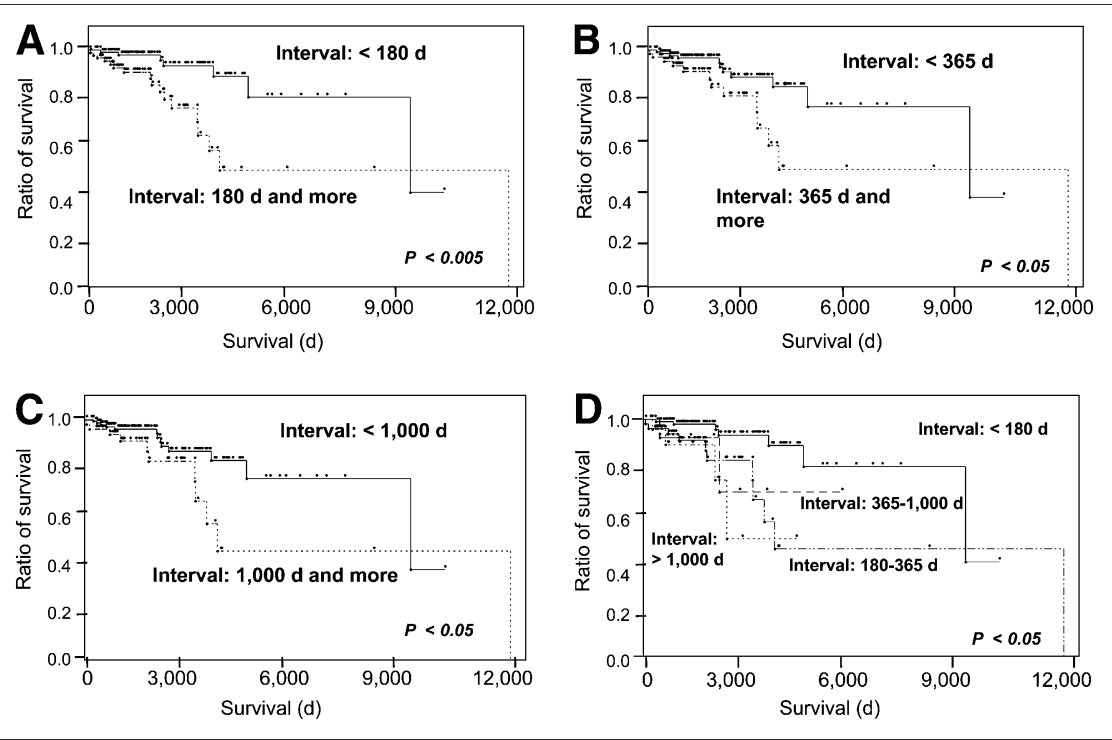

FIGURE 2. Survival curves of DTC patients analyzed by Kaplan-Meier life tables, focusing on interval between total thyroidectomy and initial RIT: $180 \mathrm{~d}(\mathrm{~A}), 365 \mathrm{~d}(\mathrm{~B}), 1,000 \mathrm{~d}$ (C), and all intervals (D). 
TABLE 2

Multivariate Analysis of Prognostic Factors for Disease-Specific Survival

\begin{tabular}{|c|c|c|c|c|c|c|c|}
\hline \multirow[b]{2}{*}{ Variable } & \multirow[b]{2}{*}{ Value } & \multicolumn{3}{|c|}{ Total } & \multicolumn{3}{|c|}{$\geq 45$ y old } \\
\hline & & $\mathrm{HR}$ & $95 \% \mathrm{Cl}$ & $P$ & $\mathrm{HR}$ & $95 \% \mathrm{Cl}$ & $P$ \\
\hline Grade of WBS at initial RIT & Fair (2) or poor (3) & 16.2 & $4.19-62.7$ & $<0.001$ & 7.22 & $2.46-21.2$ & $<0.001$ \\
\hline Metastasis extent at initial RIT & Bone metastases or more & 3.93 & $1.38-11.1$ & 0.010 & 4.39 & $1.50-12.9$ & 0.007 \\
\hline Prethyroglobulin at initial RIT & $\geq 125 \mathrm{ng} / \mathrm{mL}$ & 0.14 & $0.0001-94.6$ & 0.526 & 2.14 & $0.15-30.6$ & 0.574 \\
\hline Peak thyroglobulin at initial RIT & $\geq 1,000 \mathrm{ng} / \mathrm{mL}$ & 1.49 & $0.17-12.9$ & 0.717 & 1.27 & $0.14-11.5$ & 0.830 \\
\hline Postthyroglobulin at initial RIT & $\geq 500 \mathrm{ng} / \mathrm{mL}$ & 22.7 & $0.03-15,657$ & 0.349 & 1.88 & $0.20-17.8$ & 0.581 \\
\hline $\begin{array}{l}\text { Interval from total thyroidectomy } \\
\text { to initial RIT }\end{array}$ & $\geq 180 \mathrm{~d}$ & 4.22 & $1.55-11.5$ & 0.005 & 2.64 & $1.00-7.00$ & 0.005 \\
\hline
\end{tabular}

${ }^{*}$ Multivariable analyses were adjusted for baseline age (cutoff, $45 \mathrm{y}$ ), grade of WBS, extent of metastasis, prethyroglobulin, peak thyroglobulin, postthyroglobulin, and interval from total thyroidectomy to initial RIT

$\mathrm{HR}=$ hazard ratio; $\mathrm{Cl}=$ confidence interval.

The risk of death in patients treated by initial RIT more than $180 \mathrm{~d}$ after total thyroidectomy was 4.22 times higher than that in patients treated by initial RIT within $180 \mathrm{~d}$. This would be startling for most Japanese nuclear medicine physicians; according to a survey at a recent conference (7), about $50 \%$ of RIT centers in Japan replied that their DTC patients have to wait more than $180 \mathrm{~d}$ for RIT after referral from a surgeon. In a prognostic analysis of longterm outcome of patients with DTC, Mazzaferri et al. showed that delay in surgical therapy for more than a year after initial manifestation has an adverse effect on outcome (8). In a prognostic analysis of DTC patients with distant metastases, Schlumberger et al. showed that the survival rate was significantly improved by the early discovery of distant metastases when WBS and serum thyroglobulin measurements were routinely used (9). However, to the best of our knowledge, there has been no report showing the prognostic value of early performance of RIT after total thyroidectomy.

The present study suggests the possibility that a certain percentage of patients may experience deterioration in clinical condition because of a 180-d delay in RIT. To elucidate the medical reasons for the prognostic value of early RIT, we compared clinical data between the earlytreatment group (initial RIT within $180 \mathrm{~d}$ after total thyroidectomy) and the delayed-treatment group ( $\geq 180 \mathrm{~d}$ ) (Supplemental Table 3). The results showed no significant difference in clinical stage between the 2 groups. In addition, there was no significant difference in the average number of RITs or in sex, extent of metastasis, past history of thyroid surgery, or thyroglobulin levels. The difference in the longest blank period is conjectured to be a main reason for the poor prognosis of the delayed-treatment group. There was also a significant difference in history of other therapy between initial thyroidectomy and initial RIT. In the delayed-treatment group with other therapy $(n=28)$, most patients were treated by repeated local surgery after initial thyroidectomy and then finally developed distant metastasis $(n=24)$. It is speculated that repeated local surgery delayed the initial RIT after total thyroidectomy. The difference in grade of uptake at WBS after initial RIT may have a prognostic role. However, it is speculated that this is not because of biased patient selection but because of the delay in RIT. Delayed RIT may result in poor accumulation of radioiodine at initial RIT, probably because of latent progression of metastatic disease during the prolonged interval. Survival of DTC patients was not affected by whether hemithyroidectomy or subtotal thyroidectomy was performed before total thyroidectomy or the interval between initial thyroidectomy and total thyroidectomy.

The nonmedical reasons for delayed RIT after total thyroidectomy should be discussed. First, the long waiting list for admission to the RIT medical ward is a main reason. Recent data from our outpatient clinic showed that the delay in initial RIT for referred patients did not become remarkable until the last 3-4y, when there was a rapid increase in the number of patients who wished to receive RIT. A second reason for delayed RIT is delayed referral by the surgeons who perform total thyroidectomy. In the present study, the timing of referral to our unit was determined by the surgeons. Although more than half our patients $(n=108)$ received RIT less than $180 \mathrm{~d}$ after total thyroidectomy (Table 1$)$, the average interval between initial RIT and total thyroidectomy was $935 \pm 1,743 \mathrm{~d}$ (range, 25-10,172 d). Thus, there was a certain percentage of patients who had an extremely long interval before they were referred by their surgeons, consequently increasing the average length of the interval. This group consisted mainly of patients who developed cervical lymph node recurrence repeatedly but were treated only by local resection and finally developed unresectable disease. Shigematsu et al. showed in their surveillance report that the treatment strategy for DTC patients in Japan has been quite different from that in Europe and North America, especially in the indication for total or near-total thyroidectomy followed by RIT (10).

We also need to discuss the other significant independent prognostic factors: age at initial RIT, grade of ${ }^{131}$ I uptake on WBS, and extent of metastasis. All these factors are 
already known as independent prognostic variables for survival of DTC patients, as shown in several literature reports $(3,9,11,12)$. These reports may support the statistical credibility of our results. As was already shown in the clinical TNM staging system by UICC, age at total thyroidectomy is a significant prognostic factor for DTC patients (6). In the present study, our analysis used age at initial RIT; however, our data basically agree with the previously published data. Extent of metastasis is of course an important prognostic factor. On the one hand, the role of lymph node metastasis in prognosis is still controversial (3), but on the other hand, the presence of distant metastases is clearly a significant prognostic factor in DTC patients. Several reports using multivariate analysis have revealed that the extent of metastasis has an independent prognostic value $(9,11-13)$. The grade of ${ }^{131}$ I uptake on WBS was reported as one of the most important prognostic factors in all DTC patients, in those DTC patients with lung metastases, and in those DTC patients with bone metastases $(9,11-15)$. Serum thyroglobulin level has been reported as a useful prognostic factor for recurrence after RIT ablation, and its measurement is included as key in the follow-up management of DTC patients according to the guidelines of the British Thyroid Association and the American Thyroid Association $(1,2)$. However, most published scoring systems, including the TNM system, do not include serum thyroglobulin level as a prognostic factor. Thyroglobulin may be useful only at a specific stage of disease, especially at early stages. Our data seem to be compatible with previously published reports.

Our study had some limitations. Because it was retrospective and included only those DTC patients treated by RIT in a single unit in Japan, the results might be derived from a biased patient group. Prognostic values might have been different if the analysis had been performed on the entire group of DTC patients treated by total or near-total thyroidectomy. Many previously published prognostic analyses focused on the entire group of DTC patients who received total thyroidectomy (3). A second limitation is that our statistical analysis focused on clinical factors at the time of initial RIT, because standardization of each clinical factor in each patient with different frequencies of RIT is quite difficult. RIT is usually performed multiple times during long periods in most patients, and these cumulative therapeutic effects may have a great impact on prognosis. Further research evaluating the cumulative therapeutic effects of RIT on prognostic value in DTC patients is needed.

\section{CONCLUSION}

Our findings showed that the delay of initial RIT until more than $180 \mathrm{~d}$ after total thyroidectomy in DTC patients with metastases resulted in poor survival, whereas survival was not affected by the performance of hemithyroidectomy or subtotal thyroidectomy before total thyroidectomy or by the interval between initial thyroidectomy and total thyroidectomy. Performing initial RIT within $180 \mathrm{~d}$ after total thyroidectomy may improve survival in postoperative DTC patients with metastases.

\section{REFERENCES}

1. Watkinson JC; British Thyroid Association. The British Thyroid Association guidelines for the management of thyroid cancer in adults. Nucl Med Commun. 2004;25:897-900.

2. Cooper DS, Doherty GM, Haugen BR, et al; American Thyroid Association (ATA) Guidelines Taskforce on Thyroid Nodules and Differentiated Thyroid Cancer. Revised American Thyroid Association management guidelines for patients with thyroid nodules and differentiated thyroid cancer. Thyroid. 2009;19:1167-1214.

3. Schlumberger M, Pacini FM. Thyroid Tumors. 3rd ed. Paris, France: Nucleon; 2006.

4. ICRP publication 94: release of patients after therapy with unsealed radionuclides. Ann ICRP. 2004;34.

5. Okamoto T. Radioactive iodine treatment for differentiated thyroid carcinoma in Japan. Surgery. 2010;147:432-433.

6. International Union Against Cancer. TNM Classification of Malignant Tumours. 7th ed. West Sussex, U.K.: Wiley-Blackwell; 2009.

7. Koizumi K, Kusakabe K, Okamoto T, Kanaya S, Kanaya K, et al.; Subcommittee for Thyroid Radioiodine Therapy; the Japanese Society of Nuclear Medicine in Oncology and Immunology and the Japanese Society of Nuclear Medicine. The second surveillance report on the actual operational status of medical ward environment for radioactive iodine therapy of differentiated thyroid cancers in Japan (the second survey in 2010) [in Japanese]. Kaku Igaku. 2011;48:15-17.

8. Mazzaferri EL. Long-term outcome of patients with differentiated thyroid carcinoma: effect of therapy. Endocr Pract. 2000;6:469-476.

9. Schlumberger M, Challeton C, De Vathaire F, et al. Radioactive iodine treatment and external radiotherapy for lung and bone metastases from thyroid carcinoma. J Nucl Med. 1996;37:598-605.

10. Shigematsu N, Takami H, Kubo A. Unique treatment policy for well-differentiated thyroid cancer in Japan: results of a questionnaire distributed to members of the Japanese Society of Thyroid Surgery and the International Association of Endocrine Surgeons. Endocr J. 2006;53:829-839.

11. Casara D, Rubello D, Saladini G, et al. Different features of pulmonary metastases in differentiated thyroid cancer: natural history and multivariate statistical analysis of prognostic variables. J Nucl Med. 1993;34:1626-1631.

12. Dinneen SF, Valimaki MJ, Bergstralh EJ, Goellner JR, Gorman CA, Hay ID. Distant metastases in papillary thyroid carcinoma: 100 cases observed at one institution during 5 decades. J Clin Endocrinol Metab. 1995;80:2041-2045.

13. Pelizzo MR, Boschin IM, Toniato A, et al. Papillary thyroid carcinoma: 35 -year outcome and prognostic factors in 1858 patients. Clin Nucl Med. 2007;32: $440-444$.

14. Ronga G, Filesi M, Montesano T, et al. Lung metastases from differentiated thyroid carcinoma: a 40 years' experience. Q J Nucl Med Mol Imaging. 2004;48:12-19.

15. Pittas AG, Adler M, Fazzari M, et al. Bone metastases from thyroid carcinoma: clinical characteristics and prognostic variables in one hundred forty-six patients. Thyroid. 2000;10:261-268. 